\title{
Improving student understanding of vector fields in junior-level E\&M
}

\author{
Bert C. Xue, Ryan L. C. Hazelton, Peter S. Shaffer, and Paula R. L. Heron \\ Department of Physics, University of Washington, Box 351560, Seattle, WA, 98125
}

\begin{abstract}
Over the past several years, the Physics Education Group at the University of Washington has been developing tutorial worksheets for small-group sections for junior-level electrodynamics courses. We have observed that most of our students enter these courses with a working knowledge of static electric and magnetic fields in simple systems. However, many students have significant difficulties in transferring this knowledge to other vector fields. We first attempted to address these difficulties by having students draw analogies from basic static fields to new vector fields, but this strategy proved to be challenging for many students. Our subsequent attempt focused on familiarizing students with how the divergence and curl of a vector field defines that vector field. This approach is proving successful in enabling students to then relate this knowledge of general vector fields to understand newly introduced vector fields.
\end{abstract}

\section{INTRODUCTION}

The focus of this study is on improving student understanding of vector fields in electromagnetism (E\&M) at the junior-level. Many vector fields are introduced in E\&M (e.g., magnetic vector potential, auxiliary field, displacement field, dynamic electric field) as well as the differential forms of Maxwell's equations. One major conceptual theme present throughout E\&M is how the divergence and curl of a vector field defines that vector field. The research question of this article is: How can we improve student understanding of newly introduced vector fields in junior-level electromagnetism?

Students entering junior-level E\&M have been exposed to basic electric and magnetic fields at the introductory level. We observed that most students are able to describe simple systems (e.g., electric field of point charges, magnetic field of wires and solenoids), although they have difficulty transferring this knowledge to new fields.

Students at the University of Washington (UW) are expected to take mathematical methods courses as prerequisites to junior-level E\&M. In these courses, students are exposed to vector derivatives and associated theorems and integrals. They are expected to carry out the operations mathematically, but often enter junior-level E\&M with limited conceptual understanding of how to interpret vector derivatives $[1,2]$.

We address the research question by investigating how the conceptual framework of the curriculum affects student understanding of the magnetic vector potential $\mathbf{A}$ as a case study. When introduced in magnetostatics, $\mathbf{A}$ is analogous to the magnetic field $\mathbf{B}$ by choice of the Coulomb gauge, as both $\mathbf{A}$ and $\mathbf{B}$ have curl but no divergence. $\mathbf{A}$ is thus a prime example for investigating how students transfer their knowledge of $\mathbf{B}$ from introductory physics to new vector fields.

\section{CONTEXT FOR RESEARCH}

At UW, the junior-level E\&M courses are taught with three hours a week of traditional lectures, using Griffiths' Introduction to Electrodynamics as the textbook [3]. The courses also has 50-minute weekly tutorial sections. Each section has 14-30 students, who work in groups of 3-5 on tutorial worksheets first developed at the University of Colorado at Boulder (CU-Boulder), then at UW $[4,5]$. Two to three graduate teaching assistants supervise each tutorial. Tutorial homework is assigned weekly in addition to the lecture homework. The midterm and final exams also include an individual question based on the tutorials. Online tutorial pretests are given weekly before the tutorial session, incentivized by participation points. There are no laboratory sections for the junior-level E\&M courses.

This paper presents data from the pretest, homework, and exam questions for the Magnetic vector potential tutorial, as well as informal classroom observation. Pretests consist of multiple choice questions paired with short answer prompts for explanation. Both homework and exam questions are free response and explicitly ask for explanation.

\section{ORIGINAL APPROACH: TEACHING BY ANALOGY}

Our initial method of teaching magnetic vector potential relied on the analogous vector derivatives of $\mathbf{A}$ and $\mathbf{B}$ to help students transfer their knowledge of $\mathbf{B}$ to understanding $\mathbf{A}$. The theme of this approach is to have students explicitly use the analogy: $\mathbf{A}$ is to $\mathbf{B}$ as $\mathbf{B}$ is to $\mathbf{J}$ (the current density).

\section{A. Description of curriculum}

The initial implementation directly used CU-Boulder's tutorial. In the first section of this tutorial, students derive the curl of $\mathbf{A}$ from the divergence of $\mathbf{B}$. Students then determine the current distribution that would create a 
cylindrical region of uniform $\mathbf{B}$ along the axis (a solenoid) for practice with curl. Then, students are given the analogous vector derivatives of $\mathbf{A}$ and $\mathbf{B}$, and asked to graph (1) $\mathbf{B}$ given a uniform current-carrying wire and (2) A given the magnetic field of a solenoid, side-by-side. The second part of the tutorial involves further practice with $\mathbf{B}$ and $\mathbf{A}$ in the context of a toroidal current, and mathematically calculating $\mathbf{A}$ for a sheet of current.

When we first implemented CU-Boulder's version, our students had significant difficulty understanding why the analogy works and how to use it. We observed that most students were unable to produce analogous graphs for $\mathbf{A}$ (though they had little difficulty with $\mathbf{B}$ ), and required a great deal of TA intervention in order to apply the analogy to determine A. Few students completed more than half of the tutorial in the allotted amount of time.

We partially attribute the difference between our students' performance to CU-Boulder's expectations (as documented in their instructors' manual) to a difference in class structure. The tutorial of CU-Boulder is designed to be an integral component of a transformed curriculum, which incorporates other forms of interactive engagement aimed at promoting conceptual learning. However, physics faculty at UW choose to use a traditional lecture format for the juniorlevel E\&M, while allowing the use of tutorials to supplement lectures. It seems that our students require more scaffolding and conceptual framework than present in CU-Boulder's tutorials. We tested this hypothesis by using the CUE at the end of the course [6]. UW students performed on par with the average traditional instruction (52\%), which was significantly worse compared to the multi-institutional average of $65 \%$ using CU-Boulder's transformed curriculum [7]. This suggests that CU-Boulder's tutorials may not be well suited for use at UW, as the structure at UW requires the tutorials to be self-contained in conceptual teaching.

Our first adaptation of the E\&M tutorials continued to follow the analogy method, although with more scaffolding leading up to the analogy. This scaffolding includes asking students to apply Stokes' Theorem to the curl of $\mathbf{A}$ and presenting the analogous content for $\mathbf{B}$ first and explicitly asking students to use an analogy for A. In addition, we replaced the mathematical treatment of the sheet of current with a more conceptual treatment of the same context.

This adapted version of the tutorial seemed to be no easier for students than the initial version. Even after working through the preliminary exercises leading up to the analogy, students still required a great deal of TA intervention when asked to determine $\mathbf{A}$ for a solenoid, given B. Our assessments of student learning through pretests and posttests supported our in-class observations that many students were not able to internalize the analogy as a method to help them transfer their knowledge of $\mathbf{B}$ to understand $\mathbf{A}$.
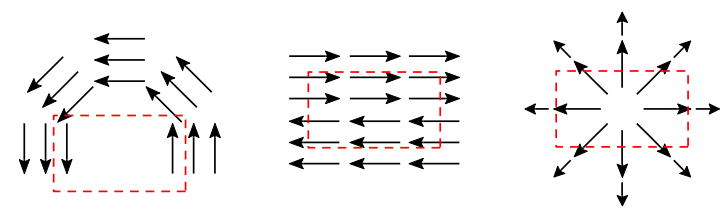

Fig 1. Vector field diagrams of A depicting (a) cylindrical curl, (b) Cartesian curl, and (c) cylindrical divergence.

\section{B. Assessment of student learning}

Our formal assessment of the tutorial involved an analysis of pretest, homework, and exam questions. The data are taken from three classes, totaling more than 120 students.

\section{Pretest: visualizing curl}

In the first part of the pretest, students were shown several vector field diagrams depicting the vector potential, with a closed boundary drawn (Fig. 1). The students chose the diagrams for which they believe a non-zero magnetic field exists. This part investigates student understanding of curl and divergence, as well as the curl relationship between $\mathbf{A}$ and $\mathbf{B}$. Additionally, students were given several possible relationships between $\mathbf{A}$ and $\mathbf{B}$ (e.g., divergence of $\mathbf{A}$ is $\mathbf{B}$, curl of $\mathbf{A}$ is $\mathbf{B}$, etc.) and asked to choose all that are true.

Fifty-two percent of the students correctly chose Fig. 1a and $64 \%$ correctly chose Fig. 1 b, but $19 \%$ incorrectly chose Fig. 1c. On the second question, $86 \%$ of the students stated that $\mathbf{A}$ and $\mathbf{B}$ were related only by curl.

\section{Pretest: analogous field}

The part of the pretest relevant to the analogy between $\mathbf{A}$ and $\mathbf{B}$ presented students with a situation in which the magnetic field behaves like the current of a thin wire (i.e., $\delta^{2}(\mathrm{~s})$ in the axial direction), and asked students to determine the direction and radial dependence (constant or dependent on s) of $\mathbf{A}$.

Sixty-one percent of the students correctly picked the azimuthal direction for $\mathbf{A}$ and a different $61 \%$ correctly answered "dependent on s," but only $42 \%$ correct on both. Only $8 \%$ answered correctly and explained explicitly by analogy, Stokes' Theorem, or a right-hand rule. Others tended to explain by stating the curl of $\mathbf{A}$ with no further interpretation or justification, which was also characteristic of incorrect answers.

\section{Homework: analogous field}

On the tutorial homework, students were asked to determine $\mathbf{B}$ given the current of a solenoid (i.e., $\delta(\mathrm{s}-\mathrm{a})$ azimuthally). Then, students were asked to describe $\mathbf{A}$ for an analogous magnetic field, and graph $\mathbf{A}$ as a function of radial distance from the axis.

Fifty-six percent of the students gave a correct description for A. An additional 5\% answered incorrectly on both questions but their answers indicated the use of analogy. 
It is interesting to note that $9 \%$ of the students incorrectly stated that $\mathbf{A}$ was zero both inside and outside the cylinder, existing only where there was $\mathbf{B}$ (i.e. the same delta function as the magnetic field). This mistake may be attributed to a misunderstanding of what an analogy is and/or the derivative nature of curl.

\section{Exam: determining vector potential}

Version 1: In one of the three classes, students were asked to consider a thick cylindrical shell with azimuthal $\mathbf{B}$ and asked to plot the three cylindrical components of $\mathbf{A}$ as a function of radial distance from the axis. Unlike the homework question, students were not given the analogous situation with current.

Of the 26 students, $27 \%$ were able to correctly identify that the vector potential only had an axial component and was non-zero at the origin, and only $15 \%$ answered with a correct dependence as well.

Version 2: In another of the three classes, students were asked to consider two infinite slabs of current and asked to determine $\mathbf{B}$, and then subsequently graph the three Cartesian components of $\mathbf{A}$ as a function of the coordinate perpendicular to the slabs. Of the 107 students, $62 \%$ correctly identified that only one component was non-zero. However, many students explicitly stated or sketched that $\mathbf{A}$ was zero when B was zero, or had qualitatively inconsistent behaviors for A. Thus, only $34 \%$ of the total can be considered qualitatively correct.

Based on the results, we concluded that teaching A solely by analogy is not enough. We decided to change our approach to teach the general framework on which the analogy is based, namely how the curl and divergence of a vector field act as sources.

\section{SUBSEQUENT APPROACH: TEACHING BY CATEGORIZATION OF FIELDS}

Our current method of teaching $\mathbf{A}$ (and several other fields) in tutorial involves helping students generalize field behavior by categorization based on their source: fields sourced by divergence but have no curl are "electrostaticlike" and fields sourced by curl but have no divergence are "magnetostatic-like." As opposed to the analogy method, this method explicitly teaches the general category first so that students can recognize that $\mathbf{B}$ is a specific case of this category, and that their knowledge of $\mathbf{B}$ can be generalized.

\section{A. Description of curriculum}

To provide the framework for the new tutorial Magnetic vector potential, we first introduce magnetostatic-like fields as a category in the prior tutorial Magnetostatic fields. Students are first asked describe and draw vector fields based on curl and divergence. Then, students are asked to reinterpret their knowledge of $\mathbf{B}$ as properties of a field sourced only by curl. After these difficult concepts have been explicitly covered generally, the framework has been established for treating a new field as a member of a category based on the field's vector derivatives.

In the new version of the Magnetic vector potential tutorial, students are first asked to review magnetostatic-like fields and Stokes' Theorem, and apply these ideas to the current-magnetic field pair to derive the familiar integral form of Ampère's law. Then, students are asked to compare the curl and divergence of $\mathbf{A}$ and $\mathbf{B}$ side-by-side. Finally, the students are asked to find the vector potential of a solenoid given the magnetic field (without first finding the magnetic field of a thick wire as was done in the previous versions).

\section{B. Assessment of student learning}

In order to assess the impact of the new version of the tutorial, we analyzed pretest, homework, and exam questions. The data are taken from one class consisting of more than 110 students. The pretest of the Magnetic vector potential tutorial for this version was identical to that described in section III, although it was given two weeks after the Magnetostatic field tutorial. The homework question (regarding $\mathbf{B}$ of a solenoid and the analogous $\mathbf{A}$ ) for this version differed by minor wording and formatting changes.

\section{Pretest: visualizing curl}

For identifying whether the sketched vector potential diagrams contained magnetic field, $56 \%$ of the students correctly chose Fig. 1a and $64 \%$ correctly chose Fig. 1b, but $12 \%$ incorrectly chose Fig. 1c. For relating A to B, 92\% of the students answered that $\mathbf{A}$ and $\mathbf{B}$ were related only by curl.

\section{Pretest: analogous field}

Sixty percent of the students answered correctly for the direction for $\mathbf{A}$ and a different $60 \%$ answered correctly for the radial dependence of $\mathbf{A}$, but only $40 \%$ answered correctly on both. Of the students who answered correctly, 26\% explained explicitly by analogy, Stokes' Theorem, a right-hand rule, or a mention of source.

\section{Homework: analogous field}

For this class, $57 \%$ of the students correctly described $\mathbf{A}$. An additional 6\% answered both questions incorrectly, but consistently. Only 3 students described $\mathbf{A}$ as a delta function at the surface of the cylinder.

\section{Exam: determining vector potential}

In the first part of the exam question used to assess the new tutorial, students were asked to consider a magnetized cylindrical shell with rotational and translational symmetry, and later sketch a symmetric B. The last part of the exam asked students to determine $\mathbf{A}$ at the axis of the cylinder, 
based on their sketch of $\mathbf{B}$. The majority of the students sketched $\mathbf{B}$ azimuthally (which was a correct answer).

Of the 96 students who drew B azimuthally, 71\% were able to correctly determine that $\mathbf{A}$ along the axis was nonzero and had a consistent axial direction with their sketch. Students with axial, radial, or no $\mathbf{B}$ were deemed correct if they correctly explained why $\mathbf{A}$ was zero at the axis. If the entire class is considered, $65 \%$ of the students were able to determine a consistent $\mathbf{A}$ for their sketch of $\mathbf{B}$.

\section{DISCUSSION}

\section{A. Comparison of performance}

There is no significant difference between the two groups of students regarding the pretest and homework data. Comparing the categorization group to the analogy group, $6 \%$ more students (from $86 \%$ to $92 \%$ ) could verbally identify that the curl of $\mathbf{A}$ is $\mathbf{B}\left(\chi^{2} \mathrm{p}=0.16\right)$, and $7 \%$ less (from $19 \%$ to $12 \%)$ incorrectly chose a divergence field $\left(\chi^{2} \mathrm{p}=0.12\right)$. There is no difference in performance on the homework questions asking students to determine $\mathbf{A}$ from $\mathbf{B}$ (around 60\%). It is interesting to note that students who are explicitly taught to use analogy do no better than students of the categorization group even though the homework explicitly presents them with analogous questions.

However, a major difference between the two groups can be seen in the exam scores. For the classes taught using the analogy method, students did rather poorly (around 30\%) when not explicitly prompted with an analogy to B. For the class taught using the categorization method, the majority of the students $(65 \%)$ are able to produce a qualitatively correct answer without being prompted to use an analogy to $\mathbf{B}$.

This difference is most notable when comparing homework and exam performance within each group. For the analogy group, students were able to identify and use an analogy when presented with analogous homework questions (around 60\%), but performance on the exam question dropped noticeably (around 30\%) when they were not prompted to think via analogy. However, students in the categorization group performed equally well on the homework and the exam, even though the analogy is explicit in the homework but not prompted in the exam. We interpret the marked difference between the two groups as evidence of an overall stronger conceptual knowledge of curl due to students having gone through the magnetostatic-like categorization tutorial.

[1] R.L.C. Hazelton, Ph.D. thesis, University of Washington, 2015

[2] C. Baily and C. Astolfi, PERC Proceedings (2014)

[3] D. Griffiths, Introduction to Electrodynamics, $4^{\text {th }}$ Ed. (Prentice-Hall, Upper-Saddle River NJ, 2012)

[4] http://www.colorado.edu/sei/departments/physics_331 0.htm
TABLE I. Method comparison.

\begin{tabular}{|c|c|c|}
\hline Question & $\begin{array}{l}\text { Analogy } \\
(\mathrm{N} \approx 130)\end{array}$ & $\begin{array}{c}\text { Categorization } \\
(\mathrm{N} \approx 110)\end{array}$ \\
\hline \multicolumn{3}{|l|}{ Pretest } \\
\hline$\nabla \times \boldsymbol{A}=\boldsymbol{B}$ & $86 \%$ & $92 \%$ \\
\hline Chose cyl. curl & $52 \%$ & $56 \%$ \\
\hline Chose Cart. curl & $64 \%$ & $64 \%$ \\
\hline Chose cyl. div. & $19 \%$ & $12 \%$ \\
\hline \multicolumn{3}{|l|}{ Homework } \\
\hline Correct & $56 \%$ & $57 \%$ \\
\hline Consistent & $61 \%$ & $63 \%$ \\
\hline Exam & $27 \%$ & $65 \%$ \\
\hline
\end{tabular}

\section{B. Implications for instruction}

In order to make a successful analogy between $\mathbf{A}$ and $\mathbf{B}$, students must identify that the vector derivatives are key characteristics in determining the field and then leverage the similarities between the vector derivatives of the two fields. This implicitly requires proficiency with treating curl and divergence of a field as sources of a field. However, student use of analogy for the example in this paper was unsuccessful. Most students seem to learn how to use the analogy when explicitly presented with an analogous pair, but fail to apply an analogy when the pair is not given. Instead, most of our students attempted to use their mathematical knowledge of curl to determine $\mathbf{A}$, although with mistakes that they would not make when determining $\mathbf{B}$ conceptually.

We have evidence, however, that teaching generally by the categorization of vector fields explicitly targets the concepts of curl and divergence, which the analogy method seems to have circumvented. This alternate method may be useful in offering students deeper explanations when exploring how divergence and curl define a field, as it allows students to compare their general knowledge of divergence and curl to their specific knowledge of electrostatic and magnetic fields. We will continue to examine how teaching fields through the categorization method can deepen students' conceptual understanding of vector derivatives and make it easier for students to learn new vector fields.

\section{ACKNOWLEDGEMENTS}

We would like to acknowledge CU-Boulder for letting us use their tutorials and the instructors who graciously allowed us to work in their classes.

[5] The Physics Education Group at the University of Washington, Tutorials in Physics: Electrodynamics. (Unpublished)

[6] S. Pollock and S. Chasteen, Colorado Upper Division Electrostatics (CUE) Assessment, (2009), <http://www.compadre.org/Repository/document/Serv eFile.cfm?ID=12653\&DocID=3226>

[7] B. Wilcox (private communication) 